Conclusion (1) A distinct subset of miRNAs is deregulated in the mucosa of actively inflamed sigmoid UC in patients who are on no treatment. (2) miR-31 and -223 are constitutionally expressed in sigmoid UC and could offer a potential diagnostic tool for patients who have no active inflammation at the time of endoscopy. (3) Manipulating miRNA expression offers promise as a potential new therapeutic pathway in active disease. (4) When investigating miRNA profiles and function it is essential to use an accurately phenotyped and homogeneous patient group. (5) We are the first to show a miRNA profile for sigmoid UC in treatment naive patients.

Competing interests None declared.

\section{OC-165 NO INCREASE IN SURGICAL COMPLICATION IN PATIENTS TREATED WITH RESCUE THERAPY FOR ACUTE SEVERE ULCERATIVE COLITIS: DATA FROM THE UK IBD AUDIT}

doi:10.1136/gutjnl-2012-302514a.165

${ }^{1} \mathrm{R}$ W Lynch, ${ }^{*} \mathrm{C}$ Down, ${ }^{2} \mathrm{M}$ Roughton, ${ }^{1,2} \mathrm{D}$ Arnott, UK IBD Audit Steering Group. ${ }^{1} \mathrm{GI}$ Unit, Western General Hospital, Edinburgh, UK; ${ }^{2}$ CEEu, Royal College of Physicians, London, UK

Introduction High dose steroids followed by colectomy if required remains the mainstay of treatment for active ulcerative colitis (UC). Recently we have seen the introduction of second-line medical therapies with the hope of avoiding the need for surgery when medical therapy fails. Using a cohort of 2981 and 3049 ulcerative colitis patients from the 2 nd and 3rd rounds of the UK IBD audit we aim to assess whether the use of second-line medical therapies has an impact on the need for surgery and complication of surgery when needed.

Methods We audited 3049 patients with ulcerative colitis. Median age was 42; there were 1421 females and 1628 males. There were 495 elective admission for surgery and 2504 acute admissions of which 882 were considered to have severe disease. Of the 2504 acute admissions; 157 underwent surgery during their admission. 202 Sites audited a median of 18 UC patients per site that were admitted with IBD between 1 September 2009 and 31 August 2010. The results of this were compared against the results from the 2008 UK IBD audit which collected data on 2981 UC patients of which there were 863 with severe disease. Of the severe patients 163 patients underwent surgery. 209 sites audited a median of 17 UC patients per site that were admitted with IBD between 1 September 2007 and 31 August 2008. Results There was no significant change in the operative rate among ASUC patients, $18.9 \%$ (163/863) in 2008 and 17.8\% (157/882) in 2010 $(p<0.6)$. Additionally there was no significant change in the mortality within the surgical populations $2.5 \%(4 / 163)$ in 2008 vs1.9\% (3/157) in 2010 ( $p<0.96)$. There were significantly more patients proceeding to surgery following anti-TNF $\alpha$ therapy in $201010.82 \%(17 / 157)$ vs $3.7 \%(6 / 163)(p<0.008)$. Using the Travis criteria we also found that there were significantly less high risk surgical patients in $2010,67.5 \%$ $(106 / 157)$ compared to $83.4 \%(136 / 163)(p<0.002)$; this was also reflected in a significant reduction in the overall amount of high risk patients in the ASUC population. Post-operative complications are not statistically different between patients who did and did not receive rescue medical therapy $40.0 \%(26 / 65)$ vs $34.8 \%(32 / 92)(p<0.8)$. There was also no statistical difference in complications between rounds, $32.5 \%(53 / 163)$ in 2008 vs $36.9 \%(58 / 157)$ in $2010(p<0.4)$.

Conclusion The surgical rate has remained the same over the two audit periods; however there has been a significant reduction in the proportion of high risk patients undergoing operations. This could in part be related to the increased use of second line medical therapies between the two audit rounds, with significantly more patients receiving anti-TNF $\alpha$ prior to surgery. The use of second line medical therapies did not increase the risk of surgery.

Competing interests None declared.

\section{OC-166 PREDICTIVE FACTORS OF DISEASE RELAPSE FOLLOWING THIOPURINE WITHDRAWAL FOR SUSTAINED CLINICAL REMISSION OF IBD}

doi:10.1136/gutjnl-2012-302514a.166

N A Kennedy, ${ }^{*}$ C J Gambles, R M Musy, G R Jones, I D Arnott, J Satsangi, C W Lees. Gastrointestinal Unit, Molecular Medicine Centre, Western General Hospital, Edinburgh, UK

Introduction Thiopurine therapy is effective in maintaining clinical remission in IBD. However, long-term therapy is associated with an increased risk of lymphoma; therefore in clinical practice we aim to withdraw therapy after $4-5$ years. Nevertheless, many patients will experience disease relapse within 12 months of drug withdrawal. ${ }^{1}$

Methods The aim of the present study was to retrospectively determine the relapse rate in ulcerative colitis (UC) and Crohn's disease (CD) following azathioprine (AZA) or mercaptopurine (MP) withdrawal and to determine factors predictive of relapse. Patients were identified by electronic case note review of an IBD research database in Edinburgh. Major inclusion criteria were AZA and/or MP therapy for a minimum of 3 years, AZA/MP withdrawn due to sustained clinical remission no steroid therapy for 6 months prior to drug withdrawal, and minimum 12 months follow-up. The primary outcome was disease relapse requiring AZA reinitiation, steroids or colectomy within 12 months of AZA/MP withdrawal, with secondary outcome assessed at 24 months. Clinical/laboratory predictors of relapse were sought. 1826 electronic records were reviewed (865 CD and $961 \mathrm{UC}$ ). 634 were treated with a thiopurine (348 CD and 286 UC). 74 met the strict study inclusion criteria (45 CD and 29 UC).

Results CD was associated with a significantly higher risk of relapse than UC on Kaplan-Meier analysis (Abstract OC-166 figure 1, $\mathrm{p}=0.026$ ). The moderate-severe relapse rate for 12 months was $44 \%$ for CD and 14\% for UC. For 24 months, relapse rates were $60 \%$ for $\mathrm{CD}$ and $48 \%$ for UC. Elevated platelet count $(p=0.03)$ and elevated white cell count $(p=0.03)$ were predictive of relapse for UC, while no predictive factors were identified for CD. Median (range) duration of thiopurine use was 6.2 (3.4-18.7) years for CD and 6.0 (3.118.0) years for UC. Median duration of follow-up was 32 months for $\mathrm{CD}$ and 45 months for UC. Retreatment with a thiopurine after relapse was successful in 7/7 cases for UC and 18/24 for CD.

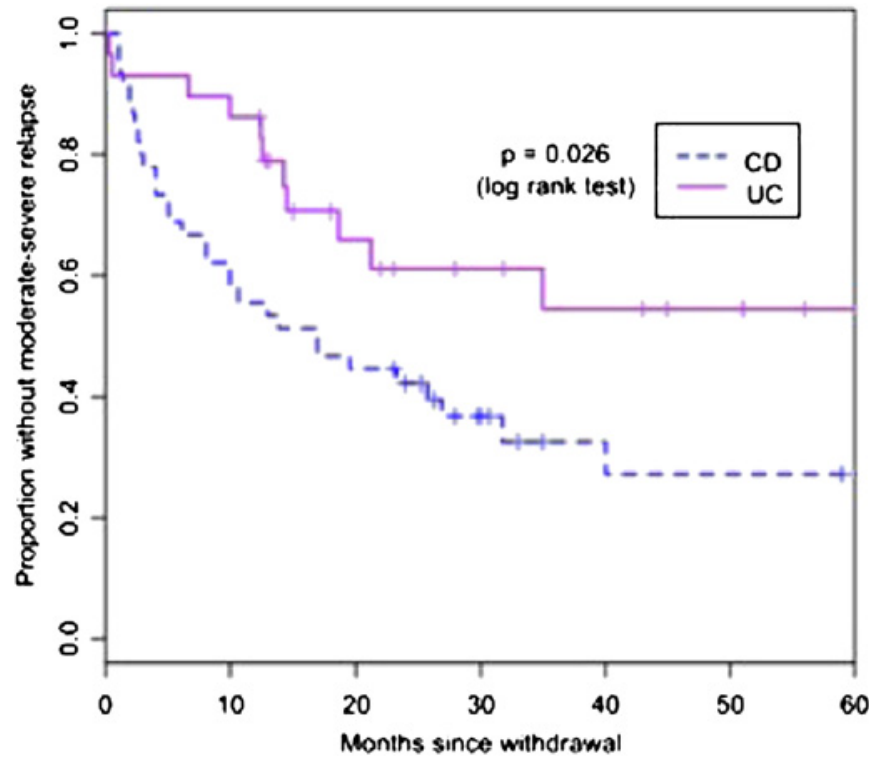

Abstract OC-166 Figure 1 Survival curves for moderate to severe relapse following thiopurine withdrawal in Crohn's disease and ulcerative colitis 